Research Article

\title{
Partnerships of Bidders with Constant Relative Risk Aversions
}

\author{
Pingping Zhao, ${ }^{1}$ Kaili Xiang, ${ }^{1}$ and Peimin Chen $\mathbb{D}^{2}$ \\ ${ }^{1}$ School of Economic Mathematics, Southwestern University of Finance and Economics, Chengdu 611130, China
}

${ }^{2}$ Shanghai Business School, Shanghai 200235, China

Correspondence should be addressed to Peimin Chen; chenpeimin@swufe.edu.cn

Received 8 November 2019; Accepted 9 March 2020; Published 6 April 2020

Academic Editor: Kishin Sadarangani

Copyright ( 2020 Pingping Zhao et al. This is an open access article distributed under the Creative Commons Attribution License, which permits unrestricted use, distribution, and reproduction in any medium, provided the original work is properly cited.

In this paper, we study a dynamic auction for allocating a single indivisible project while different participants have different bid values for the project. When the price rises continuously, the bidders can retreat the auction and obtain the compensation by the difference between the price at retreating time and the previous bid price. The final successful bidder achieves the project and pays compensations to others. We show that the auction of bidders with constant relative risk aversion (CRRA) has a unique equilibrium. While the relative risk aversion coefficient approaches to zero, the equilibrium with CRRA bidders would approach to the equilibrium with risk-neutral bidders.

\section{Introduction}

When we need to distribute an indivisible project or inheritance to multiple people (such as a house to divorced couples or heirs), a financial problem arises. Cramton et al.[1] propose that the results of allocation depend on the bidder's attitude towards risk. When bidders are risk neutral and their personal wealths are independent random variables, the necessary and sufficient conditions for effective allocation among many partners are proved. Moreover, they prove that the only equilibrium partnership is solvable as the number of bidders increases dramatically. Under the framework of homogeneous prospectives, Morgan [2] studies the fairness of dissolving the partnership between two people. Athanassoglou et al. [3] consider how to divide the project so as to minimize the maximum loss of bidders. McAfee [4] introduces a simple mechanism ([5, 6] also study the mechanism) to describe dissolvable partnerships without considering the utility function of bidders or their value distribution. Assuming that the participants are risk averse and that both bidders tend to have constant absolute risk aversion (CARA) coefficients, McAfee studies the dissolvable partnership problem and solves the bidding function under equilibrium. The second best mechanism for given initial ownership is described in [7]. In order to maximize the sum of weighted social surplus and income, the optimal dissolution mechanism for arbitrary initial ownership is demonstrated in [8]. In addition, similar to [9], most existing studies have been established under the assumption that bidders are risk neutral or the number of bidders is only two.

In order to ensure fair distribution, we need to find an appropriate allocation mechanism to allocate indivisible projects or legacies. Matt and John [10] show a dynamic auction. In the auction, the price goes up from 0 to the value that $M-1$ bidders withdraw from bidding, and the last bidder who does not withdraw from the auction wins the project. In return, the winner pays the previous bidders a compensation equal to the difference between his/her exiting price and that of the previous bidder. Let $p_{k}$ be the price, at which the $k$ th withdrawer exits the auction. We assume that $p_{0}=0$. Matt and John [10] describe the necessary and sufficient conditions for the bidding function at the case that equilibrium is symmetric equilibrium and there are $M \geq 2$ risk neutral or CARA bidders. They prove that when absolute risk aversion coefficient tends to zero, CARA equilibrium strategy tends to risk neutral equilibrium strategy.

In the same type of research, the literature studies mostly choose two bidders to study the relationship of dissolution. However, in practical cases, dissolution problems usually occur when there are more bidders. Therefore, the balancing 
strategy of two bidders has great limitations, which cannot solve the complex situation when there are more bidders. In addition, the risk preference of bidders in the relevant literature is mainly risk neutral. This is inconsistent with the diversified risk preferences of bidders in real life. In view of this, this paper synthesizes the two shortcomings above by assuming that bidders with different project value have different risk aversion levels, and the risk aversion level is negatively correlated with the project value of bidders. Furthermore, we mainly consider the impact of the project value of two or more bidders on their risk tolerance. Moreover, this paper chooses exponential utility function with constant relative risk aversion coefficient to discuss the properties of equilibrium strategy, which is different from the absolute risk aversion coefficient selected in [10]. Absolute risk aversion coefficient cannot reflect the impact of bidders' project value on their risk tolerance. When each bidder has the same relative risk aversion coefficient, we obtain their equilibrium strategy in each round and study the relationship between the equilibrium price of multiple bidders and their relative risk aversion coefficient.

In Section 2, we briefly review the models in [10]. Because bidders with different project values usually have different risk aversion degree, we introduce the utility function of relative aversion coefficient to solve equilibrium strategy, which makes the use of equilibrium strategy more widely. In Section 3, we describe the equilibrium bidding function when there are more than two CRRA bidders and prove that when their relative risk aversion coefficient tends to zero, their strategies tend to be risk neutral equilibrium.

\section{The Mathematical Model}

Assume that there are $M \geq 2$ bidders to bid an indivisible and single project and their bidding values on the project follow an identically and independently distribution. Let $F$ be their cumulative distribution function on $[0, \bar{x}]$, where $\bar{x}$ is the upper bound of bidding values with $\bar{x}<+\infty$. For simplicity, we assume that $f \equiv F^{\prime}$ is a probability density and $f(x)>0$ on $x \in[0, \bar{x}]$. Let $X_{1}, \ldots, X_{M}$ be the bidding values to the project. We sort them in the ascending order and denote them as $Z_{1}^{(M)}, \ldots, Z_{M}^{(M)}$. Let $G_{k}^{(M)}$ and $g_{k}^{(M)}$ be the cumulative distribution function and the probability density of $Z_{k}^{(M)}$, respectively. Then, the conditional density of $Z_{k+1}^{(M)}$ under given $Z_{1}^{(M)}=z_{1}, \ldots, Z_{k}^{(M)}=z_{k}$ is

$$
\begin{aligned}
& g_{k+1}^{(M)}\left(Z_{k+1}^{(M)}=z_{k+1} \mid Z_{k}^{(M)}=z_{k}\right) \\
& =(M-k) f\left(z_{k+1}\right) \frac{\left[1-F\left(z_{k+1}\right)\right]^{M-(k+1)}}{\left[1-F\left(z_{k}\right)\right]^{M-k}},
\end{aligned}
$$

and the bidder's instantaneous probability is (see [10])

$$
\lambda_{k}^{M}(z):=g_{k+1}^{(M)}\left(Z_{k+1}^{(M)}=z \mid Z_{k}^{(M)}=z\right)=(M-k) \frac{f(z)}{1-F(z)} .
$$

In an auction, the price rises continuously from 0 to $M-1$ bidders retreating from it. The last bidder who stays in the auction wins the project. Let $p_{0}=0$ and $p_{k}$ be the price of the $k$ th retreating from the auction. In return, the $k$ th round bidder retreating from the auction will receive the compensation which equals to $\left(p_{k}-p_{k-1}\right)$ from the winner, for any $k \in\{1, \ldots, M-1\}$. Thus, the winner will pay $p_{M-1}$ for the project.

We denote $\xi=\left(\xi_{1}, \ldots, \xi_{M-1}\right)$, where $\xi_{k}\left(x ; \mathbf{p}_{k-1}\right)$ is the price of retreating from the auction in the $k$ th round of a bidder whose value is $x$, when the previous prices of retreating from the auction are $p_{1}, p_{2}, \ldots, p_{k-1}$ which satisfy $p_{1}<p_{2}<\cdots<p_{k-1}$. Here, $\mathbf{p}_{k-1}:=\left(p_{1}, \ldots, p_{k-1}\right)$.

\section{Equilibrium Strategies}

We denote the utility function of bidders by

$$
u^{\gamma}(x)=\frac{1}{1-\gamma} x^{1-\gamma},
$$

where $\gamma(\gamma>0$ and $\gamma \neq 1)$ is the index of the relative risk aversion coefficient.

As the utility function of bidders is $u^{\gamma}(x)$, the bidder who wins the project will gain the payoff $u^{\gamma}\left(x_{i}-p_{M-1}\right)=$ $(1 /(1-\gamma))\left(x_{i}-p_{M-1}\right)^{1-\gamma}\left(x_{i}\right.$ is his value $)$ and retreating from the auction in the $k$ th round will gain the payoff $u^{\gamma}\left(\xi_{k}\left(x ; \mathbf{p}_{k-1}\right)-p_{k-1}\right)=(1 /(1-\gamma))\left(\xi_{k}\left(x ; \mathbf{p}_{k-1}\right)-p_{k-1}\right)^{1-\gamma}$. The term $\xi_{k}^{\gamma}\left(x, \mathbf{p}_{k-1}\right)$ denotes the equilibrium price retreating from the auction in the round $k$ when their relative risk aversion coefficient is $\gamma$.

Before we provide and verify a useful proposition, we review two lemmas as follows. It will be used in the consequent proposition.

\section{Lemma 1.}

(i) Any increasing and differentiable symmetric equilibrium $\xi$ satisfies the differential equation:

$$
\begin{aligned}
& u^{\prime}\left(\xi_{M-1}\left(x ; \mathbf{p}_{M-2}\right)-p_{M-2}\right) \xi_{M-1}^{\prime}\left(x ; \mathbf{p}_{M-2}\right) \\
& =\left[u\left(\xi_{M-1}\left(x ; \mathbf{p}_{M-2}\right)-p_{M-2}\right)-u\left(x-\xi_{M-1}\left(x ; \mathbf{p}_{M-2}\right)\right)\right] \lambda_{M-1}^{M}(x),
\end{aligned}
$$

and for $k \in\{1,2, \ldots, M-2\}$ we have that

$$
\begin{aligned}
& u^{\prime}\left(\xi_{k}\left(x ; \mathbf{p}_{k-1}\right)-p_{k-1}\right) \xi_{k}^{\prime}\left(x ; \mathbf{p}_{k-1}\right) \\
& =\left[u\left(\xi_{k}\left(x ; \mathbf{p}_{k-1}\right)-p_{k-1}\right)-u\left(\xi_{k+1}\left(x ; \xi_{k}\left(x ; \mathbf{p}_{k-1}\right), \mathbf{p}_{k-1}\right)\right.\right. \\
& \left.\quad-\xi_{k}\left(x ; \mathbf{p}_{k-1}\right)\right] \lambda_{k}^{M}(x) .
\end{aligned}
$$

(ii) If $\xi=\left(\xi_{1}, \ldots, \xi_{M-1}\right)$ is a solution to the system of differential equations in (i), then it is an equilibrium.

Lemma 2. When bidders are risk neutral, the unique symmetric equilibrium satisfies

$$
\xi_{k}^{0}\left(x ; \mathbf{p}_{k-1}\right)=\frac{M-k}{M-k+1} p_{k-1}+\frac{1}{M-k+1} E\left[Z_{M-1}^{(M)} \mid Z_{k}^{(M)}>x>Z_{k-1}^{(M)}\right] .
$$


Lemma 2 shows the unique symmetric equilibrium when bidders are risk neutral.
Proposition 1. Suppose that bidders are CRRA with the relative risk aversion coefficient $\gamma>0$. The unique symmetric equilibrium with increasing and differentiable strategies is given by

$$
\xi_{M-1}^{\gamma}\left(x, \mathbf{p}_{M-2}\right)=p_{M-2}+\left[\int_{x}^{\bar{x}}\left(\xi_{M-1}\left(z ; \mathbf{p}_{M-2}\right)-p_{M-2}\right)^{1-\gamma} J_{M-1}^{M}(z) \mathrm{d} z+\int_{x}^{\bar{x}}\left(z-\xi_{M-1}\left(z ; \mathbf{p}_{M-2}\right)\right)^{1-\gamma} J_{M-1}^{M}(z) \mathrm{d} z\right]^{1 /(1-\gamma)}
$$

and for $k=1, \ldots, M-2$,

$$
\xi_{k}^{\gamma}\left(x, \mathbf{p}_{k-1}\right)=p_{k-1}+\left[\int_{x}^{\bar{x}}\left(\xi_{k}\left(z ; \mathbf{p}_{k-1}\right)-p_{k-1}\right)^{1-\gamma} J_{k}^{M}(z) \mathrm{d} z+(M-k) \int_{x}^{\bar{x}}\left(\xi_{k+1}\left(z ; \mathbf{p}_{k}\right)-\xi_{k}\left(z ; \mathbf{p}_{k-1}\right)\right)^{1-\gamma} J_{k}^{M}(z) \mathrm{d} z\right]^{1 /(1-\gamma)}
$$

where $J_{k}^{M}(z)=\left((1-F(z))^{M-k} f(z)\right) /\left((1-F(x))^{M-k+1}\right)$.

Proof. For $u(x)=x^{1-\gamma} /((1-\gamma)(\gamma>0, \gamma \neq 1))$ and $\gamma \in(0,1)$ or $(1,+\infty)$, we have

$$
\begin{aligned}
& \frac{\partial}{\partial x}\left(\frac{1}{1-\gamma}\left(\xi_{M-1}^{\gamma}\left(x ; \mathbf{p}_{M-2}\right)-p_{M-2}\right)^{1-\gamma}(1-F(x))^{2}\right) \\
& =\left(\xi_{M-1}^{\gamma}\left(x ; \mathbf{p}_{M-2}\right)-p_{M-2}\right)^{-\gamma}(1-F(x)) \frac{\partial \xi_{M-1}^{x}\left(x ; \mathbf{p}_{M-2}\right)}{\partial x} \\
& -\frac{1}{1-\gamma} 2(1-F(x)) f(x)\left(\xi_{M-1}^{\gamma}\left(x ; \mathbf{p}_{M-2}\right)-p_{M-2}\right)^{1-\gamma} .
\end{aligned}
$$

Using Lemma 1, we obtain

$$
\begin{aligned}
& \left(\xi_{M-1}^{\gamma}\left(x ; \mathbf{p}_{M-2}\right)-p_{M-2}\right)^{-\gamma}(1-F(x)) \frac{\partial \xi_{M-1}^{x}\left(x ; \mathbf{p}_{M-2}\right)}{\partial x} \\
& =\left[\frac{1}{1-\gamma}\left(\xi_{M-1}^{\gamma}\left(x ; \mathbf{p}_{M-2}\right)-p_{M-2}\right)^{1-\gamma}\right. \\
& \left.\quad-\frac{1}{1-\gamma}\left(x-\xi_{M-1}^{\gamma}\left(x ; \mathbf{p}_{M-2}\right)\right)^{1-\gamma}\right] f(x) .
\end{aligned}
$$

Multiplying both sides of equation (10) by $(1-F(x))$, we obtain

$$
\begin{aligned}
\left(\xi_{M-1}^{\gamma}\left(x ; \mathbf{p}_{M-2}\right)-p_{M-2}\right)^{-\gamma}(1-F(x))^{2} \frac{\partial \xi_{M-1}^{x}\left(x ; \mathbf{p}_{M-2}\right)}{\partial x} \\
=\left[\frac{1}{1-\gamma}\left(\xi_{M-1}^{\gamma}\left(x ; \mathbf{p}_{M-2}\right)-p_{M-2}\right)^{1-\gamma}\right. \\
\left.\quad-\frac{1}{1-\gamma}\left(\xi_{M-1}^{\gamma}\left(x ; \mathbf{p}_{M-2}\right)\right)^{1-\gamma}\right](1-F(x)) f(x) .
\end{aligned}
$$

Substituting equation (11) into equation (9), it follows that

$$
\begin{aligned}
& \frac{\partial}{\partial x}\left(\frac{1}{1-\gamma}\left(\xi_{M-1}^{\gamma}\left(x ; \mathbf{p}_{M-2}\right)-p_{M-2}\right)^{1-\gamma}(1-F(x))^{2}\right) \\
& =-\frac{1}{1-\gamma}\left(\xi_{M-1}^{\gamma}\left(x ; \mathbf{p}_{M-2}\right)-p_{M-2}\right)^{1-\gamma}(1-F(x)) f(x)
\end{aligned}
$$$$
-\frac{1}{1-\gamma}\left(x-\xi_{M-1}^{\gamma}\left(x ; \mathbf{p}_{M-2}\right)\right)^{1-\gamma}(1-F(x)) f(x) .
$$

In addition, we have that

$$
\begin{aligned}
& \left(\xi_{M-1}^{\gamma}\left(x ; \mathbf{p}_{M-2}\right)-p_{M-2}\right)^{1-\gamma}(1-F(x))^{2} \\
& =-\int_{0}^{x}\left[\left(\xi_{M-1}^{\gamma}\left(x ; \mathbf{p}_{M-2}\right)-p_{M-2}\right)^{1-\gamma}(1-F(z)) f(z)\right. \\
& \left.\quad+\left(z-\xi_{M-1}^{\gamma}\left(z ; \mathbf{p}_{M-2}\right)\right)^{1-\gamma}(1-F(z)) f(z)\right] \mathrm{d} z+C,
\end{aligned}
$$

where $C$ is independent of $x \in[0, \bar{x})$.

From $F(\bar{x})=1$, the following equality holds: 


$$
\begin{aligned}
C= & \int_{0}^{\bar{x}}\left[\left(\xi_{M-1}^{\gamma}\left(x ; \mathbf{p}_{M-2}\right)-p_{M-2}\right)^{1-\gamma}(1-F(z)) f(z)\right. \\
& \left.+\left(z-\xi_{M-1}^{\gamma}\left(z ; \mathbf{p}_{M-2}\right)\right)^{1-\gamma}(1-F(z)) f(z)\right] \mathrm{d} z .
\end{aligned}
$$

Then, it follows that

$$
\begin{aligned}
& \left(\xi_{M-1}^{\gamma}\left(x ; \mathbf{p}_{M-2}\right)-p_{M-2}\right)^{1-\gamma}(1-F(x))^{2} \\
& =\int_{x}^{\bar{x}}\left[\left(\xi_{M-1}^{\gamma}\left(x ; \mathbf{p}_{M-2}\right)-p_{M-2}\right)^{1-\gamma}(1-F(z)) f(z)\right. \\
& \left.\quad+\left(z-\xi_{M-1}^{\gamma}\left(z ; \mathbf{p}_{M-2}\right)\right)^{1-\gamma}(1-F(z)) f(z)\right] \mathrm{d} z .
\end{aligned}
$$

That is,

$$
\begin{aligned}
& \xi_{M-1}^{\gamma}\left(x ; \mathbf{p}_{M-2}\right)=p_{M-2}+\left[\int_{x}^{\bar{x}}\left(\xi_{M-1}^{\gamma}\left(x ; \mathbf{p}_{M-2}\right)-p_{M-2}\right)^{1-\gamma} \frac{f(z)(1-F(z))}{(1-F(x))^{2}} \mathrm{~d} z\right. \\
& \left.\left.\quad+\int_{x}^{\bar{x}}\left(z-\xi_{M-1}^{\gamma}\left(x ; \mathbf{p}_{M-2}\right)\right)^{1-\gamma} \frac{f(z)(1-F(z))}{(1-F(x))^{2}} \mathrm{~d} z\right]^{1 /(1-\gamma)}\right] .
\end{aligned}
$$

We have proved that, at the round $M-1$, bid function satisfies equation (7). We shall prove that, at the round $k<M-1$, bid function satisfies equation (8) when the round $k+1$ bid function satisfies equation (8). that

Using the same way as round $M-1$, the $k$ round satisfies

$$
\begin{aligned}
& \frac{\partial}{\partial x}\left(\frac{1}{1-\gamma}\left(\xi_{k}^{\gamma}\left(x ; \mathbf{p}_{k-1}\right)-p_{k-1}\right)^{1-\gamma}(1-F(x))^{M-k+1}\right) \\
& =\left(\xi_{k}^{\gamma}\left(x ; \mathbf{p}_{k-1}\right)-p_{k-1}\right)^{-\gamma}(1-F(x))^{M-k+1} \frac{\partial \xi_{k}^{x}\left(x ; \mathbf{p}_{k-1}\right)}{\partial x} \\
& \quad-\frac{1}{1-\gamma}(M-k+1)(1-F(x))^{M-k} f(x) \\
& \quad \cdot\left(\xi_{k}^{\gamma}\left(x ; \mathbf{p}_{k-1}\right)-p_{k-1}\right)^{1-\gamma} .
\end{aligned}
$$

Using Lemma 1, we have

$$
\begin{aligned}
& \left(\xi_{k}^{\gamma}\left(x ; \mathbf{p}_{k-1}\right)-p_{k-1}\right)^{-\gamma}(1-F(x)) \frac{\partial \xi_{k}^{\gamma}\left(x ; \mathbf{p}_{k-1}\right)}{\partial x} \\
& =\frac{1}{1-\gamma}\left(\xi_{k}^{\gamma}\left(x ; \mathbf{p}_{k-1}\right)-p_{k-1}\right)^{1-\gamma}(M-k) f(x) \\
& \quad-\frac{1}{1-\gamma}\left(\xi_{k+1}^{\gamma}\left(x ; \mathbf{p}_{k}\right)-\xi_{k}^{\gamma}\left(x ; \mathbf{p}_{k-1}\right)\right)^{1-\gamma}(M-k) f(x) .
\end{aligned}
$$

Multiplying both sides of equation (18) by $((M-k+1) /(M-k))(1-F(x))^{M-k}$, we obtain

$$
\begin{aligned}
\left(\xi_{k}^{\gamma}\left(x ; \mathbf{p}_{k-1}\right)-p_{k-1}\right)^{-\gamma}(1-F(x))^{M-k+1} \frac{\partial \xi_{k}^{x}\left(x ; \mathbf{p}_{k-1}\right)}{\partial x} \\
=\frac{1}{1-\gamma}\left(\xi_{k}^{\gamma}\left(x ; \mathbf{p}_{k-1}\right)-p_{k-1}\right)^{1-\gamma}(M-k+1)(1-F(x))^{M-k} f(x) \\
\quad-\frac{1}{1-\gamma}\left(\xi_{k}^{\gamma}\left(x ; \mathbf{p}_{k-1}\right)-\xi \xi_{k}^{\gamma}\left(x ; \mathbf{p}_{k-1}\right)\right)^{1-\gamma} \\
\quad \cdot(M-k+1)(1-F(x))^{M-k} f(x) .
\end{aligned}
$$

Substituting equation (19) into equation (17), we have

$$
\begin{aligned}
\frac{\partial}{\partial x}( & \left.\frac{1}{1-\gamma}\left(\xi_{k}^{\gamma}\left(x ; \mathbf{p}_{k-1}\right)-p_{k-1}\right)^{1-\gamma}(1-F(x))^{M-k+1}\right) \\
= & -\frac{1}{1-\gamma}\left(\xi_{k}^{\gamma}\left(x ; \mathbf{p}_{k-1}\right)-p_{k-1}\right)^{1-\gamma}(1-F(x))^{M-k} f(x) \\
& -\frac{1}{1-\gamma}\left(\xi_{k}^{\gamma}\left(x ; \mathbf{p}_{k-1}\right)-\xi_{k+1}^{\gamma}\left(x ; \mathbf{p}_{k-1}\right)\right)^{1-\gamma} \\
& \cdot(1-F(x))^{M-k} f(x) .
\end{aligned}
$$

Consequently,

$$
\begin{aligned}
& \left(\xi_{k}^{\gamma}\left(x ; \mathbf{p}_{k-1}\right)-p_{k-1}\right)^{1-\gamma}(1-F(x))^{M-k+1} \\
& =-\int_{0}^{x}\left(\xi_{k}^{\gamma}\left(z ; \mathbf{p}_{k-1}\right)-p_{k-1}\right)^{1-\gamma}(1-F(z))^{M-k} f(z) \\
& \quad+\left(\xi_{k}^{\gamma}\left(z ; \mathbf{p}_{k-1}\right)-\xi_{k+1}^{\gamma}\left(z ; \mathbf{p}_{k-1}\right)\right)^{1-\gamma}(1-F(z))^{M-k} f(z) \mathrm{d} z+C .
\end{aligned}
$$

Using $F(\bar{x})=1$, we have

$$
C=\int_{0}^{\bar{x}}\left(\xi_{k}^{\gamma}\left(z ; \mathbf{p}_{k-1}\right)-p_{k-1}\right)^{1-\gamma}(1-F(z))^{M-k} f(z)+(M-k)\left(\xi_{k}^{\gamma}\left(z ; \mathbf{p}_{k-1}\right)-\xi_{k+1}^{\gamma}\left(z ; \mathbf{p}_{k-1}\right)\right)^{1-\gamma}(1-F(z))^{M-k} f(z) \mathrm{d} z .
$$


Then, it follows that

$$
\begin{aligned}
& \left(\xi_{k}^{\gamma}\left(x ; \mathbf{p}_{k-1}\right)-p_{k-1}\right)^{1-\gamma}(1-F(x))^{M-k+1} \\
& \quad=\int_{x}^{\bar{x}}\left(\xi_{k}^{\gamma}\left(z ; \mathbf{p}_{k-1}\right)-p_{k-1}\right)^{1-\gamma}(1-F(z))^{M-k} f(z)+(M-k)\left(\xi_{k}^{\gamma}\left(z ; \mathbf{p}_{k-1}\right)-\xi_{k+1}^{\gamma}\left(z ; \mathbf{p}_{k-1}\right)\right)^{1-\gamma}(1-F(z))^{M-k} f(z) \mathrm{d} z .
\end{aligned}
$$

That is,

$$
\begin{aligned}
\xi_{k}^{\gamma}\left(x ; \mathbf{p}_{k-1}\right)= & p_{k-1}+\left[\int_{x}^{\bar{x}}\left(\xi_{k}^{\gamma}\left(x ; \mathbf{p}_{k-1}\right)-p_{k-1}\right)^{1-\gamma} \frac{f(z)(1-F(z))^{M-k}}{(1-F(x))^{M-k+1}} \mathrm{~d} z\right. \\
& +M \int_{x}^{\bar{x}}\left(\xi_{k+1}^{\gamma}\left(x ; \mathbf{p}_{k}\right)-\xi_{k}^{\gamma}\left(x ; \mathbf{p}_{k-1}\right)\right)^{1-\gamma} \frac{f(z)(1-F(z))^{M-k}}{(1-F(x))^{M-k+1}} \mathrm{~d} z \\
& \left.-k \int_{x}^{\bar{x}}\left(\xi_{k+1}^{\gamma}\left(x ; \mathbf{p}_{k}\right)-\xi_{k}^{\gamma}\left(x ; \mathbf{p}_{k-1}\right)\right)^{1-\gamma} \frac{f(z)(1-F(z))^{M-k}}{(1-F(x))^{M-k+1}} \mathrm{~d} z\right]^{1 /(1-\gamma)},
\end{aligned}
$$

which is the desired result.

Proposition 1 characterizes the equilibrium price retreating from the auction in the round $k$. We know that the equilibrium price is related only to the relative risk aversion coefficient $\gamma$, the retreating prices of previous $k-1$ rounds $\mathbf{p}_{k-1}$, the value $x$, and $M-k$ from Proposition 1 . Let $M, k$, $M^{\prime}$, and $k \prime$ satisfy $M^{\prime}-k^{\prime}=M-k \geq 1$. Then, we obtain that the dropout price in the $k$ round when there are $M$ bidder equals the dropout price in the $k$ l round when there are $M^{\prime}$ bidders. Thus, we have Corollary 1.
Corollary 1. If $M^{\prime}-k^{\prime}=M-k$ and bidders are CRRA, it holds that

$$
\xi_{k^{\prime}, M}^{\gamma}\left(x ; \mathbf{p}_{k^{\prime}-1}\right)=\xi_{k, M}^{\gamma}\left(x ; \mathbf{p}_{k-1}\right)
$$

when $p_{k^{\prime}-1}=p_{k-1}$.

Example 1. There are two auctions, for simplicity, we assume that $M=10, k=9, M^{\prime}=8$, and $k^{\prime}=7$, respectively. The conditions in Corollary 1 are met, that is, $\gamma=\gamma^{\prime}, x=x^{\prime}$, $p_{k-1}=p_{k^{\prime}-1}^{\prime}$, and $M^{\prime}-k^{\prime}=M-k$. Based on Proposition 1 , we obtain

$$
\begin{aligned}
& \xi_{k^{\prime}, M^{\prime}}^{\gamma}\left(x ; \mathbf{p}_{k^{\prime}-1}\right)=\xi_{7,8}^{\gamma}\left(x ; \mathbf{p}_{6}\right)=p_{6}^{\prime}+\left[\int_{x}^{\bar{x}}\left(\xi_{7}\left(z ; \mathbf{p}_{6}\right)-p_{6}^{\prime}\right)^{1-\gamma} J_{7}^{8}(z) \mathrm{d} z+\int_{x}^{\bar{x}}\left(z-\xi_{7}\left(z ; \mathbf{p}_{6}\right)\right)^{1-\gamma} J_{7}^{8}(z) d z\right]^{1 /(1-\gamma)}, \\
& \xi_{k, M}^{\gamma}\left(x ; \mathbf{p}_{k-1}\right)=\xi_{9,10}^{\gamma}\left(x ; \mathbf{p}_{8}\right)=p_{8}+\left[\int_{x}^{\bar{x}}\left(\xi_{9}\left(z ; \mathbf{p}_{8}\right)-p_{8}\right)^{1-\gamma} J_{9}^{10}(z) \mathrm{d} z+\int_{x}^{\bar{x}}\left(z-\xi_{9}\left(z ; \mathbf{p}_{8}\right)\right)^{1-\gamma} J_{9}^{10}(z) \mathrm{d} z\right]^{1 /(1-\gamma)} .
\end{aligned}
$$

When the existence and uniqueness of solutions are satisfied, it is easy to prove that the two equations are equal.

To present an important proposition, the following useful lemma is listed firstly.

Lemma 3. The identity

$$
\begin{aligned}
& \int_{x}^{\bar{x}} \frac{(M-k+1)[1-F(t)]^{M-k} f(t)}{[1-F(x)]^{M-k+1}} \\
& \cdot E\left[Z_{M-1}^{(M)} \mid Z_{k+1}^{(M)}>t>Z_{k}^{(M)}\right] \mathrm{d} t \\
& =\left[Z_{M-1}^{(M)} \mid Z_{k+1}^{(M)}>x>Z_{k}^{(M)}\right]
\end{aligned}
$$

holds. 
Proposition 2. For any $\gamma \in(0,1)$ or $\gamma \in(1,+\infty)$, we assume that the following functions

$$
\begin{aligned}
& \left(z-\xi_{M-1}\left(z ; \mathbf{p}_{M-2}\right)\right)^{1-\gamma} J_{M-1}^{M}(z), \\
& \left(\xi_{M-1}\left(z ; \mathbf{p}_{M-2}\right)-p_{M-2}\right)^{1-\gamma} J_{M-1}^{M}(z), \\
& \left(\xi_{k}\left(z ; \mathbf{p}_{k-1}\right)-p_{k-1}\right)^{1-\gamma} J_{k}^{M}(z), \\
& \left(\xi_{k+1}\left(z ; \mathbf{p}_{k}\right)-\xi_{k}\left(z ; \mathbf{p}_{k-1}\right)\right)^{1-\gamma} J_{k}^{M}(z),
\end{aligned}
$$

are continuous and uniformly convergent if $z \in[x, \bar{x}], 0 \leq x \leq \bar{x}$, and $k \in\{1, \ldots, M-2\}$. Suppose that bidders are CRRA with the relative risk aversion $\gamma$. Then, for $k=1, \ldots, M-1$ and $\mathbf{p}_{k-1}$, it has

$$
\lim _{\gamma \longrightarrow 0^{+}} \xi_{k}^{\gamma}\left(x ; \mathbf{p}_{k-1}\right)=\xi_{k}^{0}\left(x ; \mathbf{p}_{k-1}\right) .
$$

Proof. At first, we prove that the round $M-1$ satisfies equation (29). Namely,

$$
\begin{aligned}
& \lim _{\gamma \longrightarrow 0^{+}} \xi_{M-1}^{\gamma}\left(x ; \mathbf{p}_{M-2}\right)=\lim _{\gamma \longrightarrow 0^{+}} \\
& \quad\left[\int_{x}^{\bar{x}}\left(\xi_{M-1}^{\gamma}\left(z ; \mathbf{p}_{M-2}\right)-p_{M-2}\right)^{1-\gamma} \frac{f(z)(1-F(z))}{(1-F(x))^{2}} \mathrm{~d} z\right. \\
& \left.\quad+\int_{x}^{\bar{x}}\left(z-\xi_{M-1}^{\gamma}\left(z ; \mathbf{p}_{M-2}\right)\right)^{1-\gamma} \frac{f(z)(1-F(z))}{(1-F(x))^{2}} \mathrm{~d} z\right]^{1 / 1-\gamma}+p_{M-2} .
\end{aligned}
$$

From assumptions of this proposition, we have

$$
\begin{aligned}
\lim _{\gamma \longrightarrow 0^{+}} \xi_{M-1}^{\gamma}\left(x ; \mathbf{p}_{M-2}\right)=p_{M-2} & \\
& +\left[\int_{x}^{\bar{x}}\left(\lim _{\gamma \longrightarrow 0^{+}} \xi_{M-1}^{\gamma}\left(z ; \mathbf{p}_{M-2}\right)-p_{M-2}\right) \frac{f(z)(1-F(z))}{(1-F(x))^{2}} \mathrm{~d} z\right. \\
& \left.+\left(z-\lim _{\gamma \longrightarrow 0^{+}} \xi_{M-1}^{\gamma}\left(z ; \mathbf{p}_{M-2}\right)\right) \frac{f(z)(1-F(z))}{(1-F(x))^{2}} \mathrm{~d} z\right], \\
\lim _{\gamma \longrightarrow 0^{+}} \xi_{M-1}^{\gamma}\left(x ; \mathbf{p}_{M-2}\right)=p_{M-2} & \\
& +\left[\int_{x}^{\bar{x}}\left(z-p_{M-2}\right) \frac{f(z)(1-F(z))}{(1-F(x))^{2}} \mathrm{~d} z\right] \\
= & \frac{1}{2} p_{M-2}+\int_{x}^{\bar{x}} z \frac{f(z)(1-F(z))}{(1-F(x))^{2}} \mathrm{~d} z .
\end{aligned}
$$

Using the conditional probability density

$$
\begin{aligned}
& p_{M-1}^{(M)}\left(Z_{M-1}=z_{M-1} \mid Z_{k}^{M}>x>Z_{k-1}^{M}\right) \\
& =\frac{(M-k+1)(M-k)\left(F\left(z_{M-1}\right)-F(x)\right)^{M-k-1}\left(1-F\left(z_{M-1}\right)\right) f\left(z_{M-1}\right)}{(1-F(x))^{M-k+1}},
\end{aligned}
$$

where $x<z_{M-1}<\bar{x}$, we obtain that the conditional expectation is

$$
E\left[Z_{M-1}^{(M)} \mid Z_{M-1}^{(M)}>x>Z_{M-2}^{(M)}\right]=\int_{x}^{\bar{x}} 2 z \frac{f(z)(1-F(z))}{(1-F(x))^{2}} \mathrm{~d} z .
$$

Thus,

$$
\begin{aligned}
\lim _{\gamma \longrightarrow 0^{+}} \xi_{M-1}^{\gamma}\left(x ; \mathbf{p}_{M-2}\right) & =\frac{1}{2} p_{M-2}+\int_{x}^{\bar{x}} z \frac{f(z)(1-F(z))}{(1-F(x))^{2}} \mathrm{~d} z \\
& =\frac{1}{2} p_{M-2}+\frac{1}{2} E\left[Z_{M-1}^{(M)} \mid Z_{M-1}^{(M)}>x>Z_{M-2}^{(M)}\right] .
\end{aligned}
$$

That is,

$$
\lim _{\gamma \longrightarrow 0^{+}} \xi_{M-1}^{\gamma}\left(x ; \mathbf{p}_{M-2}\right)=\xi_{M-1}^{0}\left(x ; \mathbf{p}_{M-2}\right) .
$$

From the above, we have proved that the round $M-1$ bid function satisfies equation (29). We shall prove that for the round $k$ with $k<M-1$ the bid function also satisfies equation (29) if the round $k+1$ bid function satisfies it. That is,

$$
\begin{aligned}
& \lim _{\gamma \rightarrow 0^{+}} \xi_{k+1}^{\gamma}\left(x ; \mathbf{p}_{k}\right) \\
& \quad=\frac{M-k-1}{M-k} \lim _{\gamma \longrightarrow 0^{+}} \xi_{k}^{\gamma}\left(x ; \mathbf{p}_{k+1}\right)+\frac{1}{M-k} E\left[Z_{M-1}^{(M)} \mid Z_{k+1}^{(M)}>x>Z_{k}^{(M)}\right] .
\end{aligned}
$$

Using equation (8), we have

$$
\begin{aligned}
& \lim _{\gamma \longrightarrow 0^{+}} \xi_{k}^{\gamma}\left(x ; \mathbf{p}_{k-1}\right)=p_{k-1}+\lim _{\gamma \longrightarrow 0^{+}}\left[\int_{x}^{\bar{x}}\left(\xi_{k}^{\gamma}\left(z ; \mathbf{p}_{k-1}\right)-p_{k}\right)^{1-\gamma} \frac{f(z)(1-F(z))^{M-k}}{(1-F(x))^{M-k+1}} \mathrm{~d} z\right. \\
& \left.\quad+\int_{x}^{\bar{x}}(M-k)\left(\xi_{k+1}^{\gamma}\left(z ; \xi_{k}^{\gamma}\left(z ; \mathbf{p}_{k-1}\right), \mathbf{p}_{k-1}\right)-\xi_{k}^{\gamma}\left(z ; \mathbf{p}_{k-1}\right)\right)^{1-\gamma} \frac{f(z)(1-F(z))^{M-k}}{(1-F(x))^{M-k+1}} \mathrm{~d} z\right]^{1 /(1-\gamma)}
\end{aligned}
$$


Substituting equation (23) into equation (37), we have

$$
\begin{aligned}
& \lim _{\gamma \longrightarrow 0^{+}} \xi_{k}^{\gamma}\left(x ; \mathbf{p}_{k-1}\right)=p_{k-1}+\lim _{\gamma \longrightarrow 0^{+}}\left[\int_{x}^{\bar{x}}\left(\xi_{k}^{\gamma}\left(z ; \mathbf{p}_{k-1}\right)-p_{k}\right)^{1-\gamma} \frac{f(z)(1-F(z))^{M-k}}{(1-F(x))^{M-k+1}} \mathrm{~d} z\right. \\
& \left.\quad+\int_{x}^{\bar{x}}\left\{(M-k)\left(\frac{M-k-1}{M-k} \lim _{\gamma \longrightarrow 0^{+}} \xi_{k}^{\gamma}\left(x ; \mathbf{p}_{k-1}\right)+\frac{1}{M-k} E\left[Z_{M-1}^{(M)} \mid Z_{k+1}^{(M)}>x>Z_{k}^{(M)}\right]-\xi_{k}^{\gamma}\left(z ; \mathbf{p}_{k-1}\right)\right)^{1-\gamma} \frac{f(z)(1-F(z))^{M-k}}{(1-F(x))^{M-k+1}}\right\} \mathrm{d} z\right]^{1 / 1-\gamma} \cdot
\end{aligned}
$$

We simplify equation (38) and get that

$$
\begin{aligned}
\lim _{\gamma \longrightarrow 0^{+}} \xi_{k}^{\gamma}\left(x ; \mathbf{p}_{k-1}\right)=p_{k-1}-\int_{x}^{\bar{x}} p_{k-1} \frac{f(z)(1-F(z))^{M-k}}{(1-F(x))^{M-k+1}} \mathrm{~d} z \\
\quad+\int_{x}^{\bar{x}} E\left[Z_{M-1}^{(M)} \mid Z_{k+1}^{(M)}>z>Z_{k}^{(M)}\right] \frac{f(z)(1-F(z))^{M-k}}{(1-F(x))^{M-k+1}} \mathrm{~d} z \\
=\frac{M-k}{M-k+1} p_{k-1} \\
\quad+\int_{x}^{\bar{x}} E\left[Z_{M-1}^{(M)} \mid Z_{k+1}^{(M)}>z>Z_{k}^{(M)}\right] \frac{f(z)(1-F(z))^{M-k}}{(1-F(x))^{M-k+1}} \mathrm{~d} z .
\end{aligned}
$$

Using Lemma 3, we obtain

$$
\begin{aligned}
& \int_{x}^{\bar{x}}\left\{E\left[Z_{M-1}^{(M)} \mid Z_{k+1}^{(M)}>z>Z_{k}^{(M)}\right]\right\} \frac{f(z)(1-F(z))^{M-k}}{(1-F(x))^{M-k+1}} \mathrm{~d} z \\
& =\frac{1}{M-k+1} E\left[Z_{M-1}^{(M)} \mid Z_{k+1}^{(M)}>z>Z_{k}^{(M)}\right], \\
& \lim _{\gamma \longrightarrow 0^{+}} \xi_{k}^{\gamma}\left(x ; \mathbf{p}_{k-1}\right)=\xi_{k}^{0}\left(x ; \mathbf{p}_{k-1}\right), \quad(k<M-1) .
\end{aligned}
$$

Therefore, we obtain

$$
\lim _{\gamma \longrightarrow 0^{+}} \xi_{k}^{\gamma}\left(x ; \mathbf{p}_{k-1}\right)=\xi_{k}^{0}\left(x ; \mathbf{p}_{k-1}\right), \quad(k \leq M-1) .
$$

Proposition 2 characterizes the limit property of equilibrium bid functions.

\section{Conclusions}

In this paper, we have demonstrated that the bidders' equilibrium strategies with the power utility function. We have proved that the equilibrium is unique when they have the equal relative risk aversion coefficient and we have shown that the integral equations about the bidders' equilibrium prices retreating from the auction. We have shown that the property of the equilibrium prices is that the equilibrium prices tend to the risk neutral equilibrium prices when their absolution risk aversion coefficients tend to zero. In future, we need to solve the integral equations about the equilibrium strategies and explore the relations between the equilibrium prices and the bidders' relative risk aversion coefficient.

\section{Data Availability}

All data we used are available up to request of readers.

\section{Conflicts of Interest}

The authors declare that they have no conflicts of interest.

\section{Acknowledgments}

The work was supported by the Ministry of Education in China Project of Humanities and Social Science (no. 19YJA790094) and Fundamental Research Funds for the Central Universities, P.R. China (no. JBK20030005).

\section{References}

[1] P. Cramton, R. Gibbons, and P. Klemperer, "Dissolving a partnership efficiently," Econometrica, vol. 55, no. 3, pp. 615-632, 1897.

[2] J. Morgan, "Dissolving a partnership (un)fairly," Economic Theory, vol. 23, no. 4, pp. 909-923, 2004.

[3] S. Athanassoglou, S. Brams, and J. Sethuraman, "Minimizing regret when dissolving a partnership," 2008, http://ssrn.com/ abstract=1322066 or http://dx.doi.org/10.2139/ssrn.1322066.

[4] R. P. McAfee, "Amicable divorce: dissolving a partnership with simple mechanisms," Journal of Economic Theory, vol. 56, no. 2, pp. 266-293, 2016.

[5] M. A. de Frutos, "Asymmetric price-benefits auctions," Games and Economic Behavior, vol. 33, no. 1, pp. 48-71, 2000.

[6] W. Güth and E. van Damme, "A comparison of pricing rules for auctions and fair division games," Social Choice and Welfare, vol. 3, no. 3, pp. 177-198, 1986.

[7] H. k. Chien, "Incentive efficient mechanisms for partnership dissolution," University of Bonn, Bonn, Germany, 2007.

[8] S. Loertscher and C. Wasser, "Optimal structure and dissolution of partnerships," Theoretical Economics, vol. 14, no. 3, pp. 1063-1114, 2019.

[9] B. Moldovanu, "How to dissolve a partnership," Journal of Institutional and Theoretical Economics, vol. 158, no. 1, pp. 66-80, 2002.

[10] V. E. Matt and W. John, "Dissolving a partnership dynamically," Journal of Economic Theory, vol. 166, pp. 212-241, 2016. 